\title{
Actualización del catastro de ensamble de moluscos costero-marinos del archipiélago Diego Ramírez (56 31 'S), Chile: un refugio para la economía sustentable y conservación subantártica
}

Update of the coastal-marine mollusc assembly catalog of the Archipiélago Diego Ramírez (56 31's), Chile:

a refuge for sustainable economy and Subantarctic conservation

Sebastián Rosenfeld ${ }^{1,2,3}$, Johanna Marambio ${ }^{2,3,4}$, Cristian Aldea ${ }^{5}$, Juan Pablo Rodriguez ${ }^{2,3}$,

Fabio Mendez ${ }^{2,3}$, Claudio Gonzalez-Wevar ${ }^{3,6,7}$, Karin Gerard ${ }^{2,5}$, Tamara Contador ${ }^{8,9}$,

Roy Mackenzie ${ }^{9}$, Ricardo Rozzi $i^{3,9,10}$ \& Andrés Mansilla ${ }^{2,3}$

\section{Resumen}

El archipiélago Diego Ramírez es el último confín del continente Sudamericano y se encuentra ubicado en el Paso Drake, a 112 km al SO del Cabo de Hornos aproximadamente. Debido a su difícil acceso, la información sobre la biodiversidad marina que habita sus costas es todavía limitada. En este estudio se presenta el primer catastro del ensamble de moluscos costero-marinos en este archipiélago, basado en la expedición a isla Gonzalo y en las colectas realizadas en esta isla en la primavera del año 2016. Durante marea baja se efectuó una exhaustiva inspección y colecta de moluscos desde la zona del intermareal superior hasta $1 \mathrm{~m}$ de profundidad. Para los análisis, los registros de estas colectas se complementaron con reportes de expediciones anteriores. De esta manera se determinaron 51 taxa de moluscos. Del total de taxa identificados se informan 42 nuevos registros de 42 especies de moluscos para las islas Diego Ramírez. Este estudio corresponde a un catastro actualizado de moluscos del archipiélago Diego Ramírez. Es importante seguir investigando y monitoreando este archipiélago, que corresponde al límite sur de la Provincia Magallánica y a los últimos vestigios rocosos sudamericanos que enfrentan el Paso Drake.

Palabras clave: malacofauna, Provincia Magallanica, isla Gonzalo, biodiversidad.

\section{Abstract}

The Diego Ramírez archipelago is the last point of the South-America continent, located in the
Facultad de Ciencias, Laboratorio de Ecología Molecular, Departamento de Ciencias Ecológicas,

Universidad de Chile, Santiago.

https://orcid.org/0000-0002-4363-8018

\sebastian.rosenfeld@umag.cl

2 Laboratorio de Ecosistemas Marinos Antárticos y Subantárticos (LEMAS), Universidad de Magallanes, Casilla 113-D, Punta Arenas, Chile.

3 Instituto de Ecología y Biodiversidad (IEB) Casilla 653, Santiago, Chile.

4 Science Faculty, Universität Bremen, Bremen, Germany.

5 GAIA Antártica Universidad de Magallanes, Departamento de Recursos Naturales, Bulnes 01890, Punta Arenas, Chile.

6 Instituto de Ciencias Marinas y Limnológicas (ICML), Universidad Austral de Chile, Valdivia, Chile.

7 Centro FONDAP de Investigación en Dinámica de Ecosistemas Marinos de Altas Latitudes (IDEAL), Universidad Austral de Chile, Valdivia, Chile.

8 Laboratorio Wankara de Ecosistemas Dulceacuícolas Subantárticos y Antárticos, Universidad de Magallanes \& Instituto de Ecología y Biodiversidad (IEB), Puerto Williams, Chile.

9 Programa de Conservación Biocultural Subantártica, Parque Etnobotánico Omora, Universidad de Magallanes, Teniente Muñoz 396, Puerto Williams, Chile

10 Department of Philosophy and Religion, University of North Texas, Denton, EE.UU. 
Drake Passage $112 \mathrm{~km}$ SW of Cape Horn. Due to its difficult access, information on the marine biodiversity that inhabits its coasts is still limited. This study presents the first report of the assemblage of coastal marine mollusks in this archipelago. Samples were collected during an expedition to Gonzalo Island during the spring of 2016. During the low tide an exhaustive inspection and collection of mollusks was carried out from the upper intertidal zone to $1 \mathrm{~m}$ depth. Records of these collections were complemented with reports from previous expeditions. A total 51 mollusk taxa were identified. Among the identified taxa, 42 mollusk species were new to Diego Ramirez Islands. This work is an updated list record of mollusks of the Diego Ramírez archipelago. It is important to continue investigating and monitoring this archipelago which corresponds to the southern limit of the Magellanic Province and the last South American rocky remnants that face the Drake passage.

Key words: malacofauna, Magellanic Province, Gonzalo Island, biodiversity.

\section{INTRODUCCIÓN}

Uno de los componentes importantes y característicos de los fondos marinos son los moluscos, grupo marcadamente diversificado dentro del reino animal, con más de 70.000 especies descritas (Chapman, 2009). Particularmente, en la costa chilena se han informado alrededor 959 especies (Valdovinos, 1999) con un aumento considerable de la diversidad en el extremo sur de Chile, principalmente entre los $42^{\circ} \mathrm{S}$ y $56^{\circ} \mathrm{S}$ (Valdovinos et al. 2003). Históricamente, los moluscos han sido foco de muchas investigaciones debido a su importancia ecosistémica (Aguilera, 2011) y socio-económica (Castilla \& Defeo, 2001). Es por ello que han sido objeto de numerosas investigaciones ecológicas y socio-ecológicas, tales como estudios de dinámicas temporales (e.g., Ojeda et al. 2014.), de sucesiones intermareales (e.g., Aguilera \& Navarrete, 2007), efectos antropogénicos (Sánchez-Moyano et al. 2000), e interacciones bioculturales (Ojeda et al. 2018), entre otros. Por lo tanto, los moluscos son un interesante grupo de estudio para poder evaluar posibles cambios ecosistémicos generados por causas naturales o relacionadas con actividades humanas.

El archipiélago Diego Ramírez se encuentra ubicado en el Paso Drake a $111 \mathrm{~km}$ al SO del Cabo de Hornos, y a $80 \mathrm{~km}$ al SO del archipiélago Ildefonso. Biogeográficamente, el grupo de islas Diego Ramírez corresponde al punto más al sur de la provincia Magallánica (Spalding et al. 2007). Se encuentra al borde de la plataforma continental (Pisano, 1972, Rozzi et al. 2017) y se caracteriza por presentar una gran influencia oceánica y de exposición al oleaje (Schlatter \& Riveros, 1997, Rozzi et al. 2020, este estudio). Este archipiélago está formado por dos grupos de islotes, rocas y arrecifes, separados entre sí por una extensión de $3,7 \mathrm{~km}$. El grupo principal incluye a las islas Bartolomé y Gonzalo separadas por el canal Nodales (Pisano, 1972). Las costas de ambas islas son escarpadas y con una gran pendiente, y presentan un alto grado de erosión debido a las constantes marejadas del lugar. Desde un punto de vista oceanográfico, la columna de agua que rodea el extremo sur de la provincia Magallánica, como las costas del Parque Nacional Cabo de Hornos y las aguas al sur, se caracteriza por presentar una estructura térmica vertical cuasi homogénea debido principalmente al efecto de la mezcla vertical producto de los fuertes vientos y mareas típicos de esta zona (Valdenegro \& Silva, 2003). Al igual que la temperatura, la salinidad también presenta columnas cuasi homogéneas, debido principalmente a que los fuertes vientos y regímenes de mareas típicos de esta zona favorecen la mezcla vertical al generar una columna de agua de baja estabilidad (Valdenegro \& Silva, 2003).

Aunque el archipiélago se incluye en el área de la provincia Magallánica, la información acerca de la biota intermareal de las islas Diego Ramírez es escasa (Marambio et al. 2020, este estudio). Schlatter \& Riveros (1997) reportaron tres géneros de Antozoos, 15 taxa de moluscos, 12 familias de poliquetos, 11 taxa de crustáceos, 5 especies de equinodermos y 3 especies de peces. Es importante destacar dos particularidades de Diego Ramirez: i) desde el trabajo de Schlatter \& Riveros (1997) no ha habido nuevos antecedentes en términos de fauna de invertebrados marinos bentónicos en el archipiélago Diego Ramírez, y 
ii) que esta zona constituye un área de transición de la biodiversidad subantártica-antártica y que constituye el límite de distribución sur de varias especies de molusco, entre ellos algunos de gran valor comercial para la pesca artesanal. Es por ello que esta zona constituye un excelente lugar para la conservación y uso sustentable puesto que se constituirá en un refugio de fases tempranas de desarrollo de los ciclos biológicos y reservorio genético de las poblaciones naturales. Para contribuir al conocimiento de la biota de invertebrados y a la protección de la biota del archipiélago Diego Ramírez, este trabajo ha generado un catastro actualizado de las especies de moluscos que habitan las costas de isla Gonzalo.

\section{MATERIALES Y MÉTODOS}

El área de estudio comprendió las costas rocosas de la isla Gonzalo, perteneciente al archipiélago Diego Ramírez (56³1'16,8's; $\left.68^{\circ} 42^{\prime} 53,5^{\prime \prime} \mathrm{O}\right)$ (Fig. 1). Las costas del archipiélago Diego Ramírez se caracterizan por estar ubicadas en el extremo sur de la plataforma continental de Magallanes. Estas islas junto con los islotes Ildefonso son los últimos vestigios rocosos sudamericanos que enfrentan el Paso Drake (Schlatter \& Riveros, 1997). Las costas rocosas de estas islas se caracterizan por su gran exposición al oleaje (Schlatter \& Riveros, 1997), lo cual se ve reflejada en la gran abundancia y cobertura de poblaciones
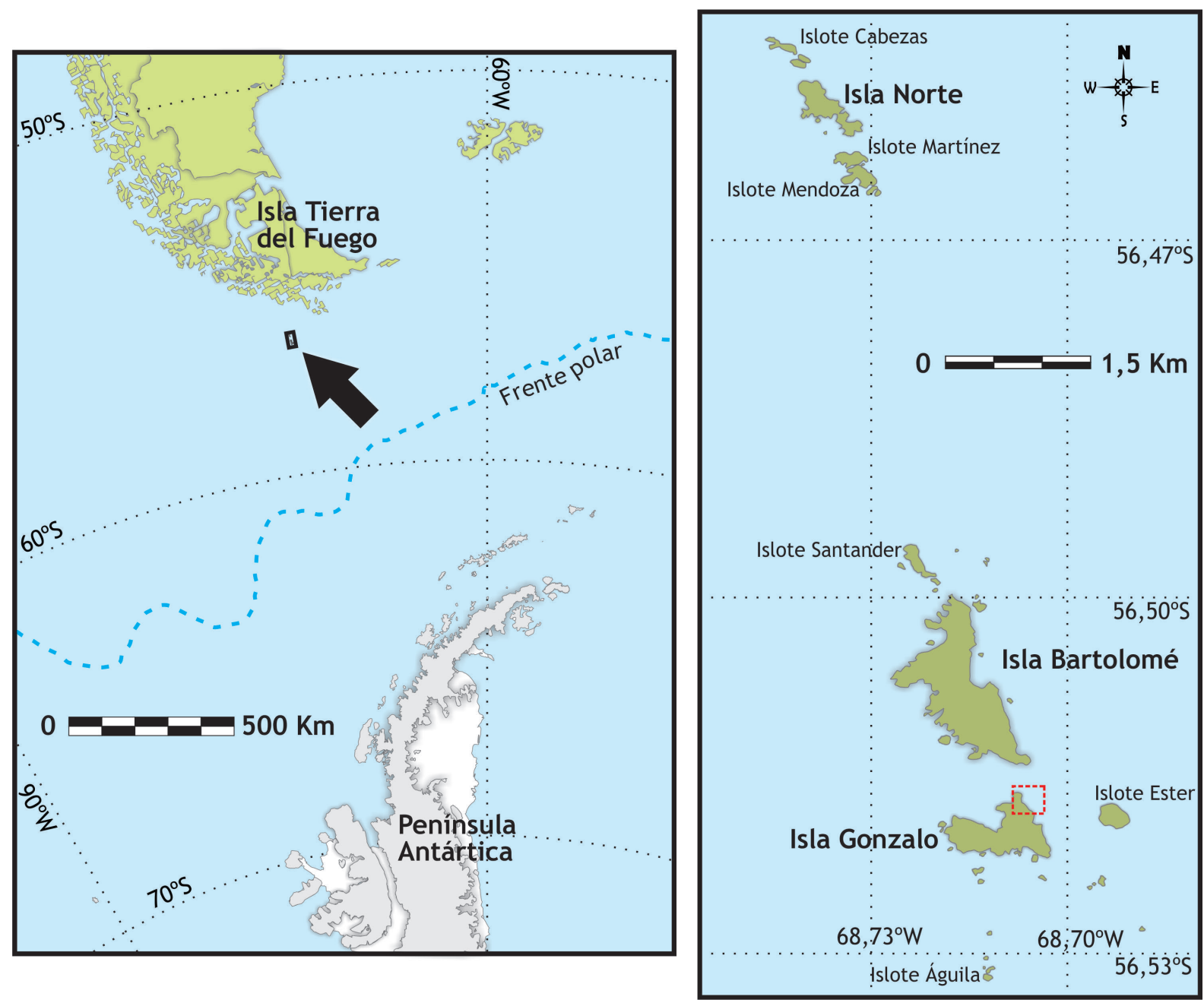

Fig. 1 Localización geográfica del archipiélago Diego Ramírez.

El recuadro en rojo señala el principal lugar de recolección en la isla Gonzalo 
del alga Durvillaea antarctica (Chamisso) Hariot. No obstante, también es posible encontrar algunas bahías protegidas ubicadas en dirección NE en las islas Bartolomé y Gonzalo (Schlatter \& Riveros, 1997). El substrato predominante presente en estas islas es el rocoso y se caracteriza por presentar una gran pendiente y un alto grado de erosión debido a las constantes marejadas del lugar. En general, en esta zona predominan los sustratos de terrazas los cuales están constituidos por grandes extensiones rocosas que pueden presentar una gran pendiente; en particular estos ambientes son considerados sitios estables para el hábitat de organismos bentónicos como moluscos y macroalgas (Rosenfeld \& Ojeda, 2015). Asimismo, se pueden encontrar ambientes inestables, por ejemplo como las playas de grava, que se encuentran en la zona de desembarco de Isla Gonzalo. La inestabilidad de estos ambientes ocasiona que sea muy difícil para los organismos bentónicos fijarse en la superficie de estos sustratos. Hasta la fecha en las costas de estas islas no se han reportado playas de fondos blandos. En general los ambientes costeros de esta zona, al igual que lo descrito para isla Hornos, están controlados principalmente por los disturbios físicos naturales como el alto hidrodinamismo producto de la exposición al oleaje (Guzmán \& Ríos, 1981; Schlatter \& Riveros, 1997).

La colecta se realizó durante la primavera del año 2016. Durante marea baja se efectuó una exhaustiva inspección visual en un transecto perpendicular a la línea de costa desde la zona del intermareal superior hasta $1 \mathrm{~m}$ de profundidad, realizando una colecta in situ de organismos vivos mediante extracción manual y espátulas. Los moluscos recolectados fueron inmediatamente puestos en bolsas plásticas rotuladas y conservados en etanol al 96\%. Los moluscos fueron identificados al nivel taxonómico más bajo posible (i.e., especie o género). Una vez identificados, los moluscos fueron puestos en frascos de vidrio con etanol al $96 \%$ para tener una colección de referencia en el laboratorio de Ecosistemas Antárticos y Subantárticos (LEMAS) de la Universidad de Magallanes.

Para la identificación taxonómica de los moluscos se utilizó la literatura más actualizada posible. Para gasterópodos y bivalvos se consultaron Reid \& Osorio (2000), Linse (2002), cotejando también con información primordial de trabajos clásicos (e.g., Smith, 1881; Rochebrune \& Mabille, 1889; Strebel, 1905a, 1905b), trabajos sistemáticos sobre taxa específicos (e.g., Zelaya, 2004; Pastorino, 2016) y bases de información académicas disponibles en internet (Morris \& Rosenberg, 2005; USNM, 2010). Como una medida de biodiversidad se calculó el índice de distinción taxonómica $\left(\Delta^{*}\right)$ por clase de molusco. Este índice tiene la particularidad de que su análisis se basa en datos cualitativos ( $\mathrm{p} / \mathrm{a}$ ) y son robustos a las diferencias de esfuerzo de muestreo (Warwick \& Clarke, 1995). Los niveles taxonómicos incluidos en este análisis fueron especie, género, familia y orden, cuyos pesos fueron: 1 (para diferentes especies), 2 (para diferentes géneros), 3 (para diferentes familias) y 4 (para diferentes órdenes).

\section{RESULTADOS}

De la campaña realizada en la isla Gonzalo se identificaron un total de 51 taxa de moluscos. En términos de riqueza por clase, Polyplacophora estuvo representada por 6 especies (6\%), Bivalvia por 13 taxa (25\%) y Gastropoda por 32 taxa, este último representando el $63 \%$ de la malacofauna identificada (Fig. 2a).

La composición taxonómica de los moluscos costeros de la isla Gonzalo se estructuró en 3 clases, 12 órdenes, 33 familias, 40 géneros representados por 51 taxa (Tabla 1). Para la clase Polyplacophora solamente se encontraron familias pertenecientes al orden Chitonida, siendo la familia Chitonidae la más representativa en términos de riqueza de especies $(\mathrm{S}=2)$, registrándose dos especies de Tonicia (Tabla 1). Para el caso de la clase Gastropoda se registraron nueve ordenes, de los cuales el orden Littorinimorpha fue el más representativo en términos de número de familias $(S=6)$ y riqueza de especies $(S=9)$. No obstante, dentro de las familias de gasterópodos la más representativa fue Nacellidae $(S=4)$ identificándose todas las especies de Nacella: N. magellanica (Gmelin 1791), N. deaurata (Gmelin 1791), N. flammea (Gmelin 1791) y $N$. mytilina (Helbling 1779) (Tabla 1), seguidas por las familias Calliostomatidae, Eatoniellidae, Muricidae $(\mathrm{S}=3$ cada una) (Tabla 1 ). En la clase Bivalvia se registraron dos órdenes, siendo Heterodonta el más representativo en número de familias $(\mathrm{S}=5)$, y 
Pteriomorphia en número de taxa $(S=7)$ (Tabla 1).

El índice de distinción taxonómica $\left(\Delta^{*}\right)$ para cada clase de moluscos arrojó valores entre 56,67 y 91,55, siendo la clase Gastropoda la que presentó el mayor valor de distinción taxonómica (Fig 2b).

\section{DISCUSIÓN}

De acuerdo a Valdovinos (1999), la costa chilena presenta alrededor de 959 especies de moluscos marinos (671 gasterópodos, 226 bivalvos y 63 poliplacóforos), incluyendo especies antárticas y de islas oceánicas. Al respecto, la provincia Magallánica $\left(41^{\circ} \mathrm{S}\right.$ a $\left.56^{\circ} \mathrm{S}\right)$ es unas de las zonas geográficas con mayor diversidad de moluscos de la costa chilena (Valdovinos et al. 2003). Utilizando la base de datos propuesta por Linse (1999) para gasterópodos y bivalvos, y Sirenko (2006) para poliplacóforos de la provincia Magallánica, se reportaron un total de 400 especies marinas (19 poliplacóforos, 250 gasterópodos y 131 bivalvos). Según dicha base de datos, los 51 taxa de moluscos registrados en este estudio corresponden al $\sim 12,75 \%$ ( 31,57 de los poliplacóforos totales, $\sim 12,8 \%$ de los gasterópodos totales y $\sim 9,92 \%$ de bivalvos totales) de los taxa citadas para estas clases. En este sentido, al comparar este valor de riqueza con otros sistemas particulares de la región como el Estrecho de Magallanes (Fig. 3), se puede observar que la riqueza de isla Gonzalo (51 taxa) es alta, considerando que sólo se han podido muestrear dos puntos de toda la isla. Por ejemplo, a lo largo de todo el Estrecho de Magallanes se han reportado 303 especies (Aldea et al. 2020). Por lo tanto, el archipiélago Diego Ramírez presentaría el $16,8 \%$ de las especies citadas para el Estrecho de Magallanes.

A excepción de la base de datos de Sirenko (2006), los porcentajes anteriormente expresados pueden ser solamente considerados como referencia, puesto que ambos reportes (Linse, 1999; Valdovinos, 1999) incluyen desde especies intermareales a abisales y algunas especies que actualmente pueden ser consideradas sinónimos júnior de otras, luego de revisiones taxonómicas de grupos específicos (e.g., Pastorino, 2005a,b; Aranzamendi et al. 2009; Gonzalez-Wevar et al. 2011, 2017; Güller et al. 2015; Pastorino, 2016). Por consiguiente, la cantidad de especies ha variado,
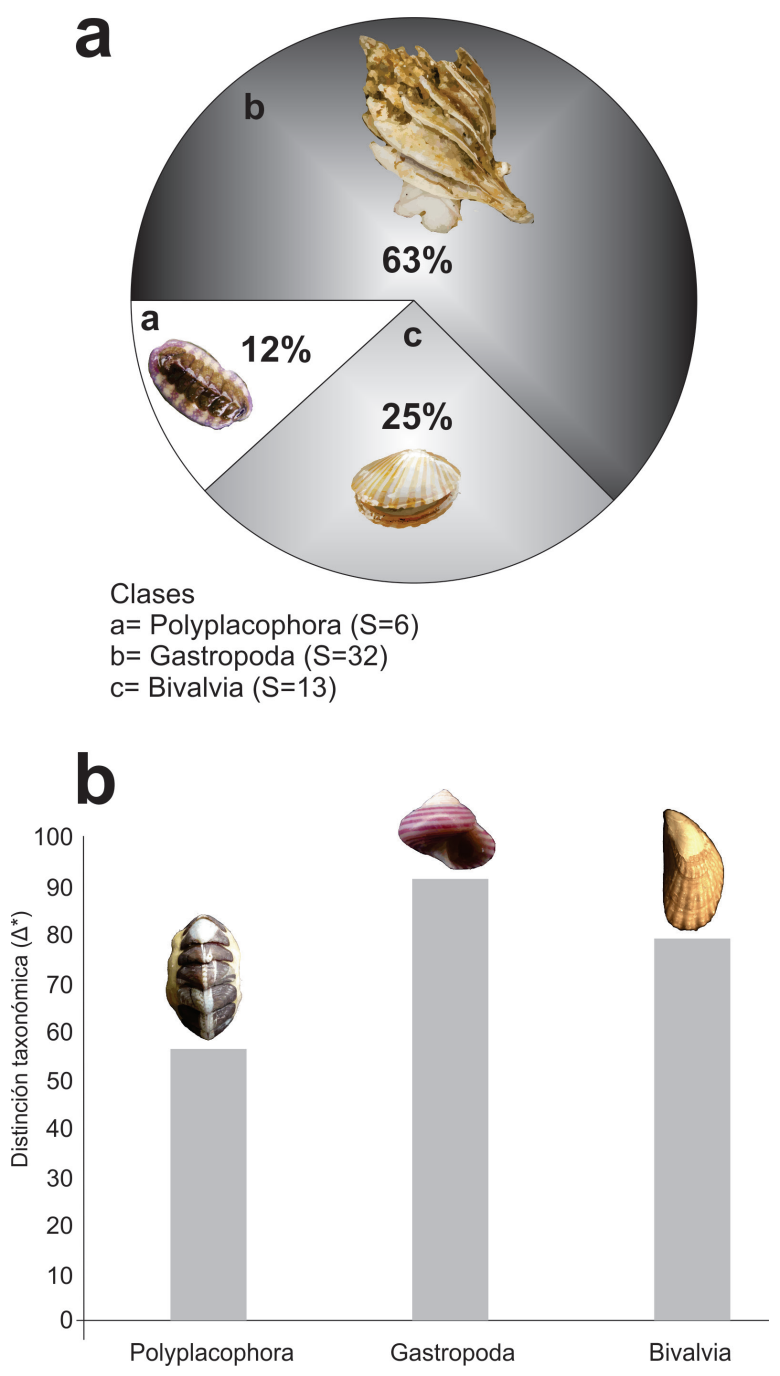

Fig. 2 A) Número de taxa de moluscos por Clase,

B) valores del índice de distinción taxonómica para cada clase de moluscos encontrados en Isla Gonzalo (Diego Ramírez) durante el año 2016.

tendiendo en algunos casos a decrecer (e.g., Zelaya \& Geiger, 2007; Gonzalez-Wevar et al. 2011). No obstante, también ha habido descripciones de nuevas especies (e.g., Güller et al. 2015; Pastorino, 2016). En este sentido, es importante seguir abordando estudios de biodiversidad en más localidades dentro de la provincia Magallánica, particularmente sitios poco visitados y que representan un gran vacío de información, como por ejemplo la zona expuesta Pacífica de los canales, el archipiélago cabo de Hornos y el archipiélago Diego Ramírez. Esta información permitiría 
Tabla. 1. Lista sistemática de todos los taxa de moluscos informados para las Islas Diego Ramírez por (3) Schlatter y Riveros (1997), (4) Valdovinos y Rüth (2005) y (*) por este estudio.

\begin{tabular}{|c|c|c|c|}
\hline \multicolumn{2}{|c|}{ Grupo: Clase a Familia } & \multirow[t]{2}{*}{ Taxa } & \\
\hline \multicolumn{3}{|l|}{ MOLLUSCA } & \\
\hline \multicolumn{4}{|l|}{ POLYPLACOPHORA } \\
\hline \multirow[t]{6}{*}{ Chitonida } & Ischnochitonidae & $\begin{array}{l}\text { Ischnochiton stramineus (Sowerby en Broderip \& } \\
\text { Sowerby, 1832) }\end{array}$ & $3, *$ \\
\hline & Callochitonidae & Callochiton puniceus (Couthouy MS, Gould, 1846) & $*$ \\
\hline & \multirow[t]{2}{*}{ Chitonidae } & Tonicia lebruni de Rochebrune, 1884 & $*$ \\
\hline & & Tonicia atrata (Sowerby, 1840) & 3 \\
\hline & Mopaliidae & Plaxiphora aurata (Spalowsky, 1795) & 3, \\
\hline & Hemiarthridae & Hemiarthrum setulosum Carpenter in Dall, 1986 & $*$ \\
\hline \multicolumn{4}{|l|}{ GASTROPODA } \\
\hline \multirow[t]{5}{*}{ Patellida } & Lottidae & Scurria ceciliana (d' Orbigny, 1841) & $*$ \\
\hline & \multirow[t]{4}{*}{ Nacellidae } & Nacella deaurata (Gmelin, 1791) & $4, *$ \\
\hline & & Nacella flammea (Gmelin, 1791) & $*$ \\
\hline & & Nacella mytilina (Helbling, 1779) & $*$ \\
\hline & & Nacella magellanica (Gmelin, 1791) & $4, *$ \\
\hline \multirow[t]{3}{*}{ Lepetellida } & \multirow[t]{2}{*}{ Fissurellidae } & Fissurella picta (Gmelin, 1791) & $*$ \\
\hline & & Fissurella oriens Sowerby, 1835 & $*$ \\
\hline & Anatomidae & Anatoma conica (d' Orbigny, 1841) & $*$ \\
\hline \multirow[t]{4}{*}{ Trochida } & Tegulidae & Tegula atra Lesson, 1832 & $*$ \\
\hline & \multirow[t]{3}{*}{ Calliostomatidae } & Margarella violacea (King \& Broderip, 1832) & $*$ \\
\hline & & Calliostoma irisans Strebel, 1905 & $*$ \\
\hline & & Calliostoma nudum (Philippi, 1845) & $*$ \\
\hline \multirow[t]{9}{*}{ Littorinimorpha } & \multirow[t]{3}{*}{ Eatoniellidae } & Eatoniella nigra (Orbigny, 1840) & $*$ \\
\hline & & Eatoniella picea Ponder \& Worsfold, 1994 & $*$ \\
\hline & & Eatoniella ebenina Ponder \& Worsfold, 1994 & $*$ \\
\hline & Littorinidae & Laevilitorina caliginosa (Gould, 1849) & $*$ \\
\hline & Newtoniellidae & Eumetula pulla (Philippi, 1845) & $*$ \\
\hline & Calyptraeidae & Crepipatella cf dilatata & $*$ \\
\hline & Rissoidae & Onoba sp. & $*$ \\
\hline & \multirow[t]{2}{*}{ Cymatiidae } & Fusitriton magellanicus (Röding, 1798) & $*$ \\
\hline & & Argobuccinum pustulosum (Lightfoot, 1786) & $*$ \\
\hline \multirow[t]{5}{*}{ Neogastropoda } & \multirow[t]{2}{*}{ Buccinidae } & Pareuthria fuscata (Bruguière, 1789) & $*$ \\
\hline & & Pareuthria atrata (E.A. Smith, 1881) & $*$ \\
\hline & \multirow[t]{3}{*}{ Muricidae } & Acanthina monodon (Pallas, 1774) & $*$ \\
\hline & & Concholepas concholepas (Bruguière, 1789) & $*$ \\
\hline & & Xymenopsis muriciformis (King \& Broderip, 1832) & $*$ \\
\hline "Lower Heterobranchia" & Mathildidae & Mathilda magellanica P. Fisher, 1783 & $*$ \\
\hline Cephalaspidea & Cylichnidae & Toledonia limnaeaeformis (E.A Smith, 1879) & $*$ \\
\hline Pteropoda & Plakobranchidae & Elysia patagonica Munian \& Ortea, 1997 & $*$ \\
\hline
\end{tabular}


Continuación Tabla 1

\begin{tabular}{|c|c|c|c|}
\hline \multicolumn{2}{|c|}{ Grupo: Clase a Familia } & \multirow[t]{2}{*}{ Taxa } & \\
\hline \multicolumn{3}{|l|}{ MOLLUSCA } & \\
\hline \multicolumn{4}{|c|}{ POLYPLACOPHORA } \\
\hline \multirow[t]{3}{*}{ Siphonariida } & Siphonariidae & Siphonaria fuegiensis Güller, Zelaya \& Ituarte, 2016 & $*$ \\
\hline & & Siphonaria lateralis (Gould, 1846) & $*$ \\
\hline & Pyramidellidae & Turbonilla strebeli Corgan, 1969 & $*$ \\
\hline \multicolumn{4}{|l|}{ BIVALVIA } \\
\hline \multirow[t]{7}{*}{ Pteriomorphia } & \multirow[t]{3}{*}{ Mytilidae } & Aulacomya atra (Molina, 1782) & $3, *$ \\
\hline & & Mytilus platensis (d'Orbigny, 1842) & $3, *$ \\
\hline & & Perumytilus purpuratus (Lamarck, 1819) & $3,{ }^{*}$ \\
\hline & Limidae & Limea pygmaea (Philippi, 1845) & $*$ \\
\hline & Pectinidae & Zygochlamys patagonica (King \& Broderip, 1832) & $*$ \\
\hline & \multirow[t]{2}{*}{ Phylobryidae } & Phylobrya sp. & $*$ \\
\hline & & Lissarca miliaris (Philippi, 1845) & $*$ \\
\hline \multirow[t]{6}{*}{ Heterodonta } & Montacutidae & Mysella sp. & $*$ \\
\hline & Neoleptonidae & Neolepton sp. & $*$ \\
\hline & Cyamiidae & Kidderia pusilla (Gould 1850) & $*$ \\
\hline & Lasaeidae & Lasaea adansoni (Gmelin, 1791) & $*$ \\
\hline & \multirow[t]{2}{*}{ Gaimardiidae } & Gaimardia trapesina (Lamark, 1819) & $3,{ }^{*}$ \\
\hline & & Gaimardia sp. & $*$ \\
\hline
\end{tabular}

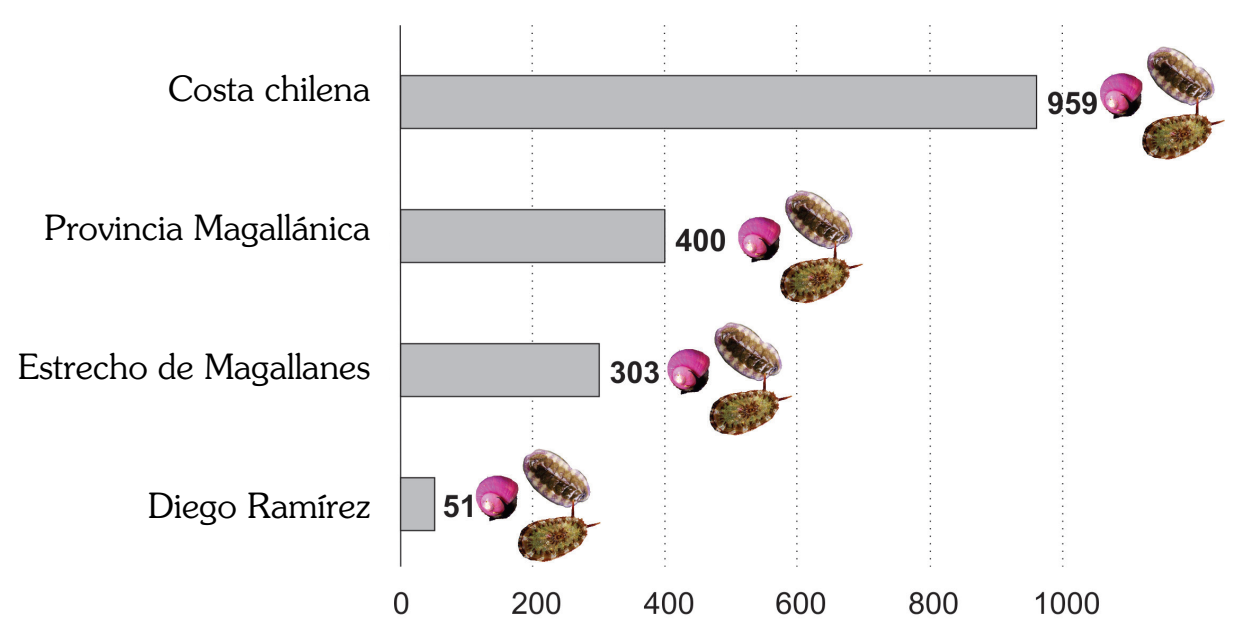

Número de especies

Fig. 3 Número de especies de moluscos citados para la costa chilena (Valdovinos, 1999), Provincia Magallanica (Linse, 1999; Sirenko, 2006), Estrecho de Magallanes (Aldea et al. 2020) e islas Diego Ramirez (este estudio).

tener un mejor conocimiento de línea base en las condiciones ecológicas contemporáneas, ello con el fin de desarrollar herramientas eficaces capaces de predecir los cambios e impactos sobre las especies y comunidades, así como para preservar los hábitats naturales (Carter, 1990).

La cantidad de especies de moluscos registrados en este trabajo representa un valor alto, 
superior al promedio de aquellas reportadas en otras investigaciones realizadas en los últimos 27 años en ambientes costeros de la ecorregión de canales y fiordos del Sur (Tabla 2). Al respecto, el trabajo que presenta el mayor número de especies (Aldea et al. 2011), reportó un total de 101 especies de moluscos, pero dicho trabajo abarcó una zona más extensiva de la micro-cuenca occidental del Estrecho de Magallanes y algunos canales adyacentes. El presente estudio se aproxima más en cantidad de especies al trabajo realizado por Rosenfeld et al. (2015a), quienes en dos localidades del Estrecho de Magallanes, registraron 42 especies asociadas a poblaciones naturales de Gigartina skottsbergii Setechell \& N.L Gardner, moluscos de los cuales el $27 \%$ de la composición del ensamble de Punta
Santa María eran micromoluscos. En este sentido, en el caso de este estudio, el $25,4 \%$ del total de taxa reportados son micromoluscos, por lo que, a pesar de que este grupo ha sido poco abordado en Magallanes, éstos podrían estar representando un alto porcentaje de la riqueza total de los ensambles bentónicos costeros. Por otra parte, Reid \& Osorio (2000) registraron 62 especies de moluscos en el sector Estero Elefante y Laguna San Rafael, ubicados en los $46^{\circ} \mathrm{S}$. Por lo tanto, al analizar el caso particular de las islas Diego Ramírez el valor de riqueza obtenido en comparación a otros trabajos no es menor, ya que, hasta la fecha se han identificado un total 51 taxa de moluscos entre los 0 y $2 \mathrm{~m}$ de profundidad siendo este reporte el cuarto con mayor riqueza (Tabla 2).

Tabla. 2. Moluscos registrados en trabajos descriptivos desde 1996 en la ecorregion de Fiordos y Canales del Sur, tomando en cuenta, estudios donde fueron recolectadas muestras intermareales y submareales

\begin{tabular}{|c|c|c|}
\hline Artículo & Latitud y Profundidad & $\mathrm{N}^{\circ}$ de taxa \\
\hline Reid \& Osorio (2000) & $45^{\circ} 38^{\prime}-46^{\circ} 37^{\prime} \mathrm{S} ; 10-15 \mathrm{~m}$ & 62 \\
\hline Linse (2002) & $52.9^{\circ}-53.7 \mathrm{~S} ; 8-522 \mathrm{~m}$ & 18 \\
\hline Ríos \& Gerdes (1997) & $52^{\circ} 30^{\prime} \mathrm{S} ; 0 \mathrm{~m}$ & 19 \\
\hline Mutschke et al. (1998) & $52^{\circ} 30^{\prime} \mathrm{S} ; 0 \mathrm{~m}$ & 27 \\
\hline Mutschke (2008) & $48^{\circ} 00^{\prime} \mathrm{S}-54^{\circ} 00^{\prime} \mathrm{S} ;$ no especifica & 25 \\
\hline Ríos et al. (2003) & $52.6 \mathrm{~S} ; 30-50 \mathrm{~m}$ & 69 \\
\hline Ríos \& Mutshke (1999) & $53^{\circ} 52^{\prime} \mathrm{S} ; 0 \mathrm{~m}$ & 17 \\
\hline Ríos et al. (2005) & $52.3^{\circ} \mathrm{S}-53.9^{\circ} \mathrm{S} ; 24-604 \mathrm{~m}$ & 15 \\
\hline Ríos et al. (2007) & $53.0^{\circ} \mathrm{S}-53.6^{\circ} \mathrm{S} ; 8 \mathrm{~m}$ & 18 \\
\hline Ríos et al. (2010) & $52.3 \mathrm{~S}^{\circ} ; 16-60 \mathrm{~m}$ & 4 \\
\hline Thatje \& Brown (2009) & $52.3^{\circ} \mathrm{S}-55.2^{\circ} \mathrm{S} ; 35-571 \mathrm{~m}$ & 21 \\
\hline Aldea \& Rosenfeld (2011) & $52^{\circ} 28^{\prime} \mathrm{S} ; 0 \mathrm{~m}$ & 12 \\
\hline Aldea et al. (2011) & $53.4^{\circ} \mathrm{S}-53.9^{\circ} \mathrm{S} ; 5-20 \mathrm{~m}$ & 101 \\
\hline Rosenfeld et al. (2013) & $53^{\circ} 37^{\prime} \mathrm{S} ; 0 \mathrm{~m}$ & 19 \\
\hline Cañete et al. (2014) & $53^{\circ} 17^{\prime} ; 0-12 \mathrm{~m}$ & 19 \\
\hline Rosenfeld et al. (2015a) & $53^{\circ} 21^{\prime} \mathrm{S}-53^{\circ}-37^{\prime} \mathrm{S} ; 10-15 \mathrm{~m}$ & 42 \\
\hline Osorio (1999) & $52.9^{\circ} \mathrm{S}-55^{\circ} 00^{\prime} ; 17-644 \mathrm{~m}$ & 38 \\
\hline Adami \& Gordillo (1999) & $54^{\circ} 00^{\prime} \mathrm{S} ; 2-10 \mathrm{~m}$ & 22 \\
\hline Ojeda et al. (2014) & $54^{\circ} 55^{\prime} \mathrm{S} ; 0 \mathrm{~m}$ & 34 \\
\hline Rosenfeld et al. (2015b) & $54^{\circ} 55^{\prime} \mathrm{S} ; 0-10 \mathrm{~m}$ & 36 \\
\hline Promedio ( \pm D.E.) & & $31 \pm 23$ \\
\hline Este reporte & $56^{\circ} 31^{\prime} \mathrm{S} ; 0-2 \mathrm{~m}$ & 51 \\
\hline
\end{tabular}


De los 51 taxa registrados en este estudio, solamente 9 habian sido reportados previamente para el archipiélago Diego Ramírez (Schlatter \& Riveros, 1997; Valdovinos \& Rüth, 2005), por lo tanto, el reporte de los otros 42 taxa correspondería al primer registro de estos moluscos para el archipiélago Diego Ramírez, específicamente la zona costera de la isla Gonzalo. En este sentido, es importante destacar que en el listado de moluscos de Diego Ramírez se registraron especies raras, como el gasterópodo Mathilda magellanica, un micromolusco muy poco conocido dentro de la provincia Magallánica debido principalmente a su escasa cantidad de registros; fue registrada únicamente por (Aldea et al. 2011) para la entrada occidental del Estrecho de Magallanes. Al respecto, esta especie no está considerada en el listado de moluscos de la región de Magallanes propuesto por (Linse, 1999).

También se pudo reportar dentro del Archipielago poblaciones de Concholepas concholepas. Se trata de un resultado relevante principalmente porque este recurso en Chile es de suma importancia para la pesca artesanal (Osorio, 2002), y su presencia en Diego Ramírez podría ser importante para futuros planes de conservación de esta especie.

Dentro de las tres clases de moluscos, Gastropoda presentó el mayor valor de distinción taxonómica $\left(\Delta^{*}\right)$, indicando que los gasterópodos someros de Diego Ramírez estarían más distanciados taxonómicamente entre sí, mientras que el bajo valor obtenido para la clase Polyplacophora $\left(\Delta^{*}\right)$ indicaría que las especies están más relacionadas entre sí. Este índice se ha utilizado en otras investigaciones, por ejemplo, en zonas de latitudes altas del Hemisferio Norte se reportó que los moluscos muestran una pequeña tendencia a aumentar el valor de $\left(\Delta^{*}\right)$ hacia el norte (Renaud et al. 2009). En este sentido, estudios realizados en la Península Antártica Oeste y Mar de Bellingshausen en comunidades de moluscos han arrojado valores similares de distinción taxonómica $\left(\Delta^{*}\right)$ (Aldea et al. 2009). Por lo tanto, el valor obtenido en este estudio es la primera aproximación del estado actual del ensamble de moluscos someros que habitan las costas del archipiélago Diego Ramírez, indicando que hacia regiones subpolares y polares del Hemisferio Sur la distinción taxonómica en moluscos podría presentar una tendencia similar a lo descrito en el Hemisferio Norte por Renaud et al. (2009).

Finalmente, es importante destacar que a pesar de los esfuerzos realizados en la región por diversos autores (ver Tabla 2), aún existe desconocimiento sobre la biodiversidad de moluscos marinos en la provincia Magallánica, y más aún en estos sitios alejados tales como el archipiélago Diego Ramírez. Es por ello muy relevante seguir investigando y monitoreando este archipiélago, que corresponde al limite sur de la provincia Magallánica y a los últimos vestigios rocosos sudamericanos que enfrentan el Paso Drake.

\section{AGRADECIMIENTOS}

Los autores quieren agradecer al Consejo Nacional de Investigación en Ciencia y Tecnología (CONICYT) del programa FONDECYT otorgado 1110875 a A.M y la Iniciativa Científica Milenio (concesión P05-002 ICM, Chile) y al programa FONDECYT 1161358 otorgado a K.G. S.R., C.GW y K.G. agradecen al proyecto PIA CONICYT ACT172065. Los autores agradecen al Instituto de Ecología y Biodiversidad de Chile a través del proyecto Centros Tecnológicos de Excelencia con Financiamiento Basal CONICYT AFB170008. A.M. agradece el apoyo de Proyecto FONDECYT 1180433. Un especial agradecimiento para la Armada de Chile, al Comandante en Jefe de la III Zona Naval, Contraalmirante Ivo Brito, al Jefe de Estado Mayor General, Vicealmirante José Miguel Rivera, al Comité Oceanográfico Nacional y a la Comandancia del Distrito Naval Beagle, como también a la tripulación de la nave OPV 83 Marinero Fuentealba, al personal de traslado en helicóptero y de logística institucional, y al personal del Faro de la isla Gonzalo por su invaluable apoyo para el desarrollo de investigación de terreno en el sitio de estudios ecológicos permanente implementado en el archipiélago Diego Ramírez.

\section{LITERATURA CITADA}

Adami, M. L., \& Gordillo, S. (1999). Structure and dynamics of the biota associated with Macrocystis pyrifera (Phaeophyta) from the Beagle Channel, Tierra del Fuego. Scientia 
Marina, 63(1), 183-191.

Aguilera, M. (2011). The functional roles of herbivores in the rocky intertidal systems in Chile: A review of food preferences and consumptive effects. Revista Chilena de Historia Natural, 84, 241-261.

Aguilera, M. A., \& Navarrete, S. A. (2007). Effects of Chiton granosus (Frembly, 1827) and other molluscan grazers on algal succession in wave exposed mid-intertidal rocky shores of central Chile. Journal of Experimental Marine Biology and Ecology, 349, 84-98.

Aldea, C., Olabarría, C., \& Troncoso, J. (2009). Spatial patterns of benthic diversity in molluscs from West Antarctica. Antarctic Science, 21(4), 341-353.

Aldea, C., \& Rosenfeld, S. (2011). Moluscos intermareales de la Playa Buque Quemado (Estrecho de Magallanes, Chile). Revista de Biología Marina y Oceanografía, 46, 115124.

Aldea, C., Rosenfeld, S., \& Cárdenas, J. (2011). Caracterización de la diversidad de moluscos bentónicos sublitorales en la isla Carlos III y áreas adyacentes, Estrecho de Magallanes, Chile. Anales del Instituto de la Patagonia, 39(2), 73-89.

Aldea, C., Novoa, L., Alcaino, S., \& Rosenfeld, S. (2020). Diversity of benthic marine mollusks of the Strait of Magellan, Chile (Polyplacophora, Gastropoda, Bivalvia): a historical review of natural history. ZooKeys, 963, 1-36.

Aranzamendi, M. C., Gardenal, C. N., Martin, J. P., \& Bastida, R. (2009). Limpets of the genus Nacella (Patellogastropoda) from the Southwestern Atlantic: species identification based on molecular data. Journal of Molluscan Studies, 75, 241-251.

Cañete, J. I., Aldea, C., Figueroa, T., Medina, A., Haro, D., Pérez, I., Rodrigo, C. \& Kathleen, H. (2014). Guía de identificación de la macrofauna bentónica de Bahía Porvenir, Chile. Punta Arenas, Chile: Universidad de Magallanes.

Carter, R. W. (1990). Coastal environments: An introduction to the physical, ecological, and cultural systems of coastlines. London: Academic Press.
Castilla, J. C., \& Defeo, O. (2001). Latin American benthic shellfisheries: emphasis on $\mathrm{co}^{-}$ management and experimental practices. Reviews in Fish Biology and Fisheries, 11, 1-30.

Chapman, A. (2009). Numbers of living species in Australia and the world. 2nd edition. Report for the Australian Biological Resources Study. Australian Government. Department of the Environment, Water, Heritage and the Arts. $80 \mathrm{pp}$.

González-Wevar, C., Nakano, T., Cañete, I., \& Poulin, E. (2011). Concerted genetic, morphological and ecological diversification in Nacella limpets in the Magellanic Province. Molecular Ecology, 20, 19361951.

Gonzalez-Wevar, C. A., Hüne, M., Segovia, N. I., Nakano, T., Spencer, H. G., Chown, S. L., Saucède, T., Johnstone, G., Mansilla, A., \& Poulin, E. (2017). Following the Antarctic Circumpolar Current: patterns and processes in the biogeography of the limpet Nacella (Mollusca: Patellogastropoda) across the Southern Ocean. Journal of Biogeography, 44, 861-874.

Güller, M., Zelaya, D., \& Ituarte, C. (2015). How many Siphonaria species (Gastropoda: Euthyneura) live in southern South America?. Journal of Molluscan Studies, 1-17, doi:10.1093/mollus/eyv036

Guzmán, L., \& Ríos, C. (1981). Estructura del conjunto de macroorganismos de una playa de bloques y cantos rodados de Isla Wollaston, Archipielago del Cabo de Hornos. Anales del Instituto de la Patagonia, 12, 258-270.

Linse, K. (1999). Mollusca of the Magellan region. A checklist of the species and their distribution. Scientia Marina, 63(1), 399407.

Linse, K. (2002). The shelled magellanic Mollusca: with special reference to biogeographic relations in the Southern Ocean. Theses Zoologicae, 34, 1-252.

Marambio, J., Rosenfeld, S., Rodríguez, J. P., Méndez, F., Contador, T., Mackenzie, R., Goffinet, B., Rozzi, R., \& Mansilla, A. (2020). Siete nuevos registros de macroalgas para el archipiélago Diego Ramírez 
(56³1's): el valor del nuevo parque marino como sumidero de carbono y conservación de la biodiversidad subantártica. Anales del Instituto de la Patagonia, 48, en revisión.

Morris, P.J., \& Rosenberg, G. (2005). Search interface and documentation for Malacolog, an online database of Western Atlantic marine Mollusks. [www database (version 4.1.1)] URL: http:// www.malacolog.org. (Consultado el 25/09/2017.).

Mutschke, E., Ríos, C., \& Montiel, A. (1998). Situación actual de la macrofauna presente en el intermareal de bloques y cantos de Bahía Laredo, Estrecho de Magallanes. Anales del Instituto de la Patagonia, 26, 5-29.

Mutschke, E. (2008). Biodiversidad y estructura de la comunidad macrobentónica en canales y fiordos australes. N. Silva, \& S. Palma. (Ed.) Avances en el conocimiento oceanográfico de las aguas interiores chilenas, Puerto Montt a Cabo de Hornos. Comité Oceanográfico Nacional - Pontificia Universidad Católica de Valparaíso, Valparaíso, 133-14.

Ojeda, J., Rosenfeld, S., Marambio, J., Rozzi, R., \& Mansilla, A. (2014). Patrones estacionales y espaciales de la diversidad de moluscos intermareales de bahía Róbalo, canal Beagle, Reserva de la Biosfera Cabo de Hornos, Chile. Revista de Biología Marina y Oceanografía, 49(3), 493-509.

Ojeda, J., Rozzi, R., Rosenfeld, S., Contador, T., Massardo, F., Malebrán, J., GonzálezCalderón, J., \& Mansilla, A. (2018). Interacciones bioculturales del pueblo Yágan con las macroalgas y moluscos: una aproximación desde la filosofía ambiental de campo. Magallania, 46(1), 155-181.

Osorio, C. 1999. Gastrópodos prosobranquios del extremo sur de Chile. Boletín del Museo Nacional de Historia Natural, Chile, 48, 37-49.

Osorio, C. (2002). Moluscos marinos en Chile: especies de importancia económica. Santiago: Facultad de Ciencias, Universidad de Chile. 211 pp.

Pastorino, G. (2005a). Recent Naticidae (Mollusca: Gastropoda) from the Patagonian Coast.
The Veliger, 47(4), 225-258.

Pastorino, G. (2005b). A revision of the genus Trophon Monfort, 1810 (Gastropoda: Muricidae) from southern South America. The Nautilus, 119(2), 55-82.

Pastorino, G. (2016). Revision of the genera Pareuthria Strebel, 1905, Glypteuthria Strebel, 1905 and Meteuthria Thiele, 1912 (Gastropoda: Buccinulidae) with the description of three new genera and two new species from Southwestern Atlantic waters. Zootaxa, 4179(3), 301-344.

Pisano, E. (1972). Observaciones Fito-ecológicas en las islas Diego Ramírez. Anales del Instituto de la Patagonia, 1-2, 161-169.

Reid, D. G., \& Osorio, C. (2000). The shallowwater marine mollusca of the Estero Elefantes and Laguna San Rafael, southern Chile. Bulletin of the Natural History Museum of London, Zoology, 66(2), 109146.

Renaud, P. E., Webb, T. J., Bjorgesaeter, A., Karakassis, I., Kedra, M., Kendall, M. A. et al. 2009. Connnetal-scale patterns in benthic invertebrate diversity: insights from the MacroBen database. Marine Ecology Progress Serie, 382, 239-252.

Ríos, C., \& Gerdes, D. (1997). Ensamble bentónico epifaunístico de un campo intermareal de bloques y cantos en Bahía Laredo, Estrecho de Magallanes. Anales del Instituto de la Patagonia, 25, 47-55.

Ríos, C., \& Mutschke, E. (1999). Community structure of intertidal boulder-cobble fields in the Straits of Magellan, Chile. Scientia Marina, 63(1), 193-201.

Ríos, C., Mutschke, E., \& Morrison, E. (2003). Biodiversidad bentónica sublitoral en el estrecho de Magallanes, Chile. Revista de Biología Marina y Oceanografía, 38(1), 1-12.

Ríos, C., Mutschke, E., Montiel, A., Gerdes, D. \& Arntz, W.E. (2005). Soft-bottom macrobenthic faunal associations in the southern Chilean glacial fjord complex. Scientia Marina, 69(2), 225-236.

Ríos, C., Arntz, W., Gerdes, D., Mutschke, E., \& Montiel, A. (2007). Spatial and temporal variability of the benthic assemblages 
associated to the holdfasts of the kelp Macrocystis pyrifera in the Straits of Magellan, Chile. Polar Biology, 31, 89100.

Ríos, C., Mutschke, E. \& Montiel, A. (2010). Estructura de la comunidad macrofaunística bentónica en la boca oriental del Estrecho de Magallanes, Chile austral. Anales del Instituto de la Patagonia, 38(1), 83-96.

Rochebrune, A. T., \& Mabille, J. (1889). Mollusques. Mision Scientifique du Cap Horn. VI, Zoologie, Paris, 129 pp.

Rosenfeld, S., Marambio, J., \& Aldea, C. (2013). Comparación de ensambles de moluscos en dos sustratos intermareales de la cuenca central del Estrecho de Magallanes. Amici Molluscarum, 21(2), 7-18.

Rosenfeld, S., Aldea, C., Mansilla, A., Marambio, J., \& Ojeda, J. (2015a). Richness, systematics, and distribution of molluscs associated with the macroalga Gigartina skottsbergii in the Strait of Magellan, Chile: A biogeographic affinity study. Zookeys, 519, 49-100.

Rosenfeld, S., Aldea, C., Ojeda, O., Hüne, M., Marambio, J., \& Mansilla, A. (2015b). Habitantes marinos. En T. Contador, S. Rosenfeld, J. Ojeda, \& J. Kennedy. (Eds.) Historia natural de los invertebrados del Cabo de Hornos (pp. 104-176). Punta Arenas: Chile Fundación Omora, Universidad de Magallanes.

Rosenfeld, S., \& Ojeda, O. (2015). Habitanteshábitats y hábitos marinos. En T. Contador, S. Rosenfeld, J. Ojeda, \& J. Kennedy. (Eds.) Historia natural de los invertebrados del Cabo de Hornos (pp. 40-51). Punta Arenas: Chile Fundación Omora, Universidad de Magallanes.

Rozzi, R., Massardo, F., Mansilla, A., Squeo, F. et al. (2017). Parque Marino Cabo de Hornos - Diego Ramírez. Punta Arenas, Chile: Ediciones Universidad de Magallanes.

Rozzi, R., Crego, R. D., Contador, T. Schüttler, E., Rosenfeld et al. (2020). Un centinela para el monitoreo del cambio climático y su impacto sobre la biodiversidad en la cumbre austral de América: la nueva red de estudios socioecológicos a largo plazo Cabo de Hornos. Anales del Instituto de la Patagonia, 48, en revisión.

Sánchez-Moyano, J. E., Estacio F. J., GarcíaAdiego, E. M., \& García-Gómez, J. C. (2000). The molluscan epifauna of the alga Halopteris scoparia in southern Spain as a bioindicator of coastal environmental conditions. Journal of Molluscan Studies, 66, 431-448.

Schlatter, R.P., \& Riveros, G.M. (1997). Historia natural del Archipiélago Diego Ramírez, Chile. Serie Científica INACH, 47, 87112.

Sirenko, B. (2006). Report on the present state of our knowledge with regard to the chitons (Mollusca: Polyplacophora) of the Magellan Strait and Falkland Islands. Venus, 65(1-2), 81-89.

Smith, E. A. (1881). Account of the Zoological collections made during the survey of the H.M.S. "Alert" in the Straits of Magellan and on the coast of Patagonia. IV. Mollusca and Molluscoidea. Proceedings of the Zoological Society of London, 1881, 22-44.

Spalding, M. D., Fox, H. E., Allen, G. R., Davidson, N., Ferdaña, Z. A., Finlayson, M., Halpern, B. S., Jorge, M. A., Lombana, A., Lourie, S. A., Martin, K. D., Mcmanus, E., Molnar, J., Recchia, C. A., \& Robertson, J. (2007). Marine ecoregions of the world: A bioregionalization of coastal and shelf areas. BioScience, 57(7), 573-583.

Strebel, H. (1905a). Beiträge zur Kenntnis der Molluskenfauna der Magalhaen-Provinz, II. Die Trochiden. Zoologische Jahrbücher, 8, 121-166.

Strebel, H. (1905b). Beiträge zur Kenntnis der Molluskenfauna der Magalhaen-Provinz. No 3. Zoologische Jahrbücher, 22, 575-666.

USNM. (2010). National Collection of the Smithsonian National Museum of Natural History, USNM (http://invertebrates. si.edu/). Consultado el 3/05/2018.

Thatje, S. \& Brown, B. (2009). The macrobenthic ecology of the Straits of Magellan and the Beagle Channel. Anales del Instituto de la Patagonia, 37(2), 17-27.

Valdenegro, C., \& Silva, N. (2003). Caracterización oceanográfica física y química de la zona de canales y fiordos australes de chile entre el 
estrecho de Magallanes y Cabo de Hornos (Cimar 3 fiordos). Ciencia y Tecnología del Mar, 26(2), 19-60.

Valdovinos, C. (1999). Biodiversidad de moluscos chilenos: Base de datos taxonómica y distribución. Gayana, 63(2), 111-164.

Valdovinos, C., \& Rüth, M. (2005). Nacellidae limpets of the southern end of South America: taxonomy and distribution. Revista Chilena de Historia Natural, 78, 497-517.

Valdovinos, C., Navarrete, S., \& Marquet, P. (2003). Mollusk species diversity in the Southeastern Pacific: why are there more species towards the pole? Ecography, 26,
139-144.

Warwick, R. M., \& Clarke, K. R. (1995). New 'biodiversity' measures reveal a decrease in taxonomic distinctness with increasing stress. Marine Ecology Progress Series, 129, 301-305.

Zelaya, D. (2004). The genus Margarella Thiele, 1893 (Gastropoda: Trochidae) in the southwestern Atlantic ocean. The Nautilus, 118(3), 112-120.

Zelaya, D., \& Geiger, D.L. (2007). Species of Scissurellidae and Anatomidae from SubAntarctic and Antarctic waters (Gastropoda: Vetigastropoda). Malacologia, 49(2), 393443. 